\title{
Labordiagnostik in der Rheumatologie
}

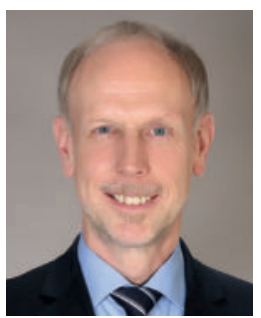

Prof. Dr. Andreas Krause
Die Labordiagnostik einschließlich infektions- und immunserologischer Verfahren ist unentbehrlicher Bestandteil der modernen rheumatologisch-immunologischen Diagnosefindung. Voraussetzung für den optimalen Einsatz der Labordiagnostik und damit für die richtige Interpretation der Ergebnisse sind jedoch genaue Kenntnisse über Präanalytik, Leistungsfähigkeit und Limitationen der einzelnen Parameter bzw. Methoden. Das vorliegende Heft widmet sich daher den wichtigsten und teils sehr komplexen Bereichen der aktuellen rheumatologischen Labordiagnostik.

R. Mierau gibt zunächst einen Überblick zur Labordiagnostik entzündlicher Gelenkerkrankungen. Dabei geht es sowohl um das sogenannte Basislabor und die allgemeine Entzündungsdiagnostik wie natürlich um Rheumafaktoren und Autoantikörper gegen citrullinierte Peptide. Komplettiert wird der Beitrag durch Hinweise zur oftmals vernachlässigten Synovia-Analyse und zur sinnvollen Bestimmung des HLA-B27.

In dem Beitrag von A. Krause wird die Anwendung und Interpretation der infektionsserologischen Diagnostik kritisch beleuchtet. Da es, abgesehen von den septischen Arthritiden, selten gelingt, den auslösenden Erreger einer Arthritis direkt nachzuweisen, ist die Labordiagnostik infektassoziierter Arthritiden in der Regel auf den Nachweis von Antikörpern gegen potenzielle Erreger beschränkt mit all ihren Limitationen und Fallgruben.

Die Bedeutung von Autoantikörpern in der Diagnostik von Kollagenosen, Myositiden und Vaskulitiden beschreibt E. Csernok in ihrem Beitrag. Schwerpunkte sind dabei die antinukleären Antikörper (ANA) und die anti-Neutrophilen zytoplasmatischen Antikörper (ANCA). Abschließend folgen einige wichtige Laborparameter zur Aktivitätsbeurteilung dieser Autoimmunerkrankungen.

Anschließend vermittelt uns V. Jansen einen tiefen Einblick in die moderne ANA-Diagnostik. Nach Beschreibung der Methodik macht sie uns mit der neuen ICAP-Nomenklatur für antinukleäre Antikörper (ANA) vertraut. In einer Tabelle werden die neuen ANA-Codes, die Zielantigene und Krankheitsassoziationen der verschiedenen ANA-Subtypen übersichtlich zusammengefasst.

Im letzten Beitrag bringen uns T. Rose und T. Dörner auf den neuesten Stand zum Antiphospholipid-Antikörpersyndrom. Dabei wird sowohl die spezielle Labordiagnostik erklärt wie auch die aus dem klinischen Verlauf und den Laborbefunden resultierenden therapeutischen Konsequenzen.

Insgesamt gibt dieses Themenheft somit einen hervorragenden, sehr aktuellen Überblick zu wichtigen Aspekten der rheumatologisch-immunologischen Labordiagnostik. Ich danke allen Autoren für ihre hervorragenden, praxisrelevanten Beiträge und wünsche allen Leserinnen und Lesern eine interessante Lektüre mit hohem Erkenntnisgewinn.

Ihr

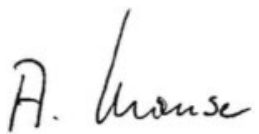

